6. Steinberg FM, Guthrie NL Villablanca AC et al. (2003): Soy protein with isoflavones has favorable effects on endothelial function that are independent of lipid and antioxidant effects in healthy postmenopausal women. Am. J. Clin. Nutr. 78, 123-130.

7. West SG, Hilpert KF, Juturu V et al. (2005): Effects of including soy protein in a blood cholesterol-lowering diet on markers of cardiac risk in men and in postmenopausal women with and without hormone replacement therapy. J. Womens Health (Larchmt). 14(3), 253-262.

8. Eugene A. Borodin, Iraida G. Menshikova, Vladimir A. Dorovskikh, Natalya A. Feoktistova et al. (2009). Effects of twomonth consumption of $30 \mathrm{~g}$ a day of soy protein isolate or skimmed curd protein on blood lipid concentration in Russian adults with hyperlipidemia. J. Nutr. Sci. Vitaminol. 55, 492-497.

9. Sacks F.M., Lichtenstein A, Van Horn L et al. (2006): Soy Protein, Isoflavones, and Cardiovascular Health An American Heart Association Science Advisory for Professionals From the Nutrition Committee. Circulation. 113, 1034-1044.

UDC 577.125:616.153.915/.922/.96:616.441-008.63:57.084 DOI 10.22448/AMJ.2017.3.32-34

\title{
THE INFLUENCE OF THE TREATMENT OF RATS WITHIN A LIFE WITH ENRICHED SOYA DIET ON LIPIDS OF BLOOD, LIVER AND BRAIN
}

\author{
Feoktistova N.A., Shtarberg M.A., Borodin E.A.
}

\author{
Amur State Medical Academy, Blagoveshchensk, Russian Federation
}

Nervous tissue is extremely rich in lipids, [18] which changes, and especially their oxidative modifi-cation [3,4], play an important role in the functioning of the brain during various stages of ontogen-esis and in particular during the aging period. [2] Vegetable oils are the main source of unsaturated lipids and fat-soluble antioxidants for the organism of animals and humans [11]. The most common vegetable oil is soy, it accounts for half of all vegetable oils produced in the world [19]. The litera-ture discusses the ability of soy products to reduce cholesterol in the blood [20] and prevent a de-cline in cognitive function in postmenopausal women [13,15]. In experiments on laboratory animals [1] and clinical studies [7], we demonstrated antioxidant [1,8] and hypocholesterolemic effects [7] from the intake of soy products. We present the results of the study of the effect of long-term soy intake on the content of some lipids and products of their oxidative modification in the blood, liver and brain of rats, and attempts are made to use them to explain the ability of soya that we have es-tablished to prevent a decrease in the cognitive abilities of rats in adulthood growth [6].

The study was performed on white laboratory rats (18 males and 24 females). The animals of the control group received a standard diet, and the experimental three days a week the standard di-et, and four days boiled soya. A detailed description of the conditions of feeding of rats was de-scribed earlier [6]. The animals were removed from the experiment at the age of 15 month. Blood plasma, erythrocyte mass, liver and brain tissue were analyzed. Biochemical parameters of blood plasma were determined with the help of standard reagent sets on the biochemical semi-automatic analyzer Stat Fax 1904+. Lipids were extracted from the erythrocyte mass, liver and brain samples of the rats using Folch method [12]. In the lipid extracts, the content of total lipids was determined gravimetrically, cholesterol, phospholipids, diene conjugates and hydroperoxides with color reac-tions [1]. UV absorption spectra of lipid extracts were recorded on a UNICO 2804 spectrophotome-ter [9]. The fatty acid composition of brain lipids in the combined extracts of the control and exper-imental groups of rats was studied by gas-liquid chromatography of methyl esters of fatty acids on the gas-liquid chromatogram Crystal 2000M, column DB-23, carrier gas-purity 0.9999. Chromato-gram analysis was performed using the Chromatec Analytic 2.5 program [5].

Feeding rats with large quantities of soy for a lifetime did not affect the rates depending on the nature of the diet, and the animals receiving the enriched diet did not differ in the content of total protein, glucose, cholesterol, triglycerides, and activity of alanine aminotransferase (ALAT) in the blood plasma with vivo-control group. The sex of the animals did not matter.

There were no differences in the content of total lipids, cholesterol, phospholipids and vitamin $E$ in erythrocytes, depending on the sex of the animals. The only changes in the lipid composition and oxidation state of the erythrocyte lipids in the experimental and control group included a mod-erate increase in cholesterol by $28 \%$ and a decrease in the content of diene conjugates also by $28 \%$ in the erythrocytes of males that received soy.

In the group of animals treated with soya a marked increase in the content of vitamin $E$ in the liver, in both females and males, was found 1.3-1.5 times, respectively, which is understanda-ble taking into account the high content of this antioxidant in the soybean used consisting of $380 \mu \mathrm{g} / \mathrm{g}$ wet weight [8]. Other changes in the lipid composition and the degree of oxidation of liver lipids in animals on soybean diet depended on sex. In particular, a statistically significant increase in the phospholipid content by $18 \%$ and an antioxidant effect, manifested in a decrease in the con-tent of diene conjugates (by $25 \%$ based on phospholipids), conjugated trienes and ketodienes (A278nm) (by $37 \%$ ) in animals, treated with soya, were characteristic only for females. The con-tent of lipid hydroperoxides in the control and experimental groups of rats was $2.81 \pm 0.4$ and $1.88 \pm 0.21 \mathrm{nmol} / \mathrm{mg}$ phospholipid, respectively, but due to the large variation in the values in the con-trol groups, the differences did not reach statistical significance. The reason for the dependence of the antioxidant effect of soya on the gender of animals may be associated with greater oxidation of liver lipids in the control group of rats in adulthood compared to males, as indicated by a higher content of conjugated trienes and ketodienes (by 40\%) and lipid hydroperoxides (1,5 times in terms of the wet weight of the liver and 2 times in terms of phospholipids) in the liver of females in relation to males. 
The results of the study of fatty acid composition of brain lipids testify to a decrease in the content of saturated palmitic and stearic acids and an increase in the content of unsaturated oleic, linolelaidinic and eicosenic acids in the animals of the experimental group, which can be as-sociated with a high content of unsaturated fatty acids in soybean oil [14]. In the functioning of the brain, an important role is played by linoleic acid, and despite its insignificant content in the li-pids of the nervous tissue [17]. Linoleic acid is considered as a neurotransmitter [10]. Given the exceptionally high content of linoleic acid in soybean oil (51-57\% of all fatty acids) [14] it could be assumed that feeding to animals during the life of soybeans would lead to an increase in the content of linoleic acid in neurolipids, but this did not happen. Linoleic acid remained a minor component in brain lipids of animals receiving soya. Therefore, it is hardly advisable to evaluate the influence of the soy diet on the fatty acid composition of brain lipids on changes in the content of linoleic acid from 0.6 to $1.1 \%$. Of some interest is the higher content in the brain lipids of males feeding on soybean, arachidonic (20:4) and docosahexaenoic (22:6) acid - precursors of biologi-cally active lipids. This is not observed in females. The content of arachidonic acid in the controls is higher than in females by $14 \%$, and docosahexaenoic by $42 \%$. When studying the UV absorp-tion spectra of lipid extracts of the brain, regardless of sex, the content of oxidized forms of lipids in the brain of animals receiving a diet enriched with soybean is lower than that of the control ones.

The results obtained show that the soybean enriched diet affected the lipid composition and their oxidative modification in the liver and brain of animals due to the ingestion of significant amounts of unsaturated lipids and fat-soluble antioxidants. Given the important role of lipids in brain function [2-4], the results of this study are consistent with the positive effect of soybean on the cognitive ability of rats at adulthood [6]. Another reason, no doubt, is the effect of isoflavo-noids contained in soybeans in significant amounts [16], but the complexity of determining their trace amounts in animal tissues does not allow us today to discuss this mechanism.

\section{References}

1. Borodin EA, Dorovskikh VA, Aksenova TV, Shtarberg MA Lipid composition and antioxi-dant properties of soy milk under in vitro and in vivo conditions // Far Eastern Medical Jour-nal, 2001, N4, P.26-30.

2. Eremenko IR, Vasilieva EV, Ryzhavsky B.Ya., Litvintseva E.M. The content of lipids in the cortex of the cerebral hemispheres of the brain and cerebellum in rats in the dairy and puber-tal period was normal and with an experimental increase in brain mass // Far-and-Slow Med-ical Journal. 2010. № 4. P. 109-111.

3. Zadvornaya OV, Lebedko OA, Ryzhavsky B.Ya. Effect of the administration of sustanon-250 to male and female rats in the pubertal period on the indices of their development and free radical oxidation in the cerebral cortex // Far Eastern Medical Journal. 2010. № 2. P. 108-111.

4. Lebedko OA, Ryzhavsky B.Ya., Zadvornaya O.V. Free-radical status of the neocortex of white rats and its modification by the derivatives of testosterone // Far-Eastern Medical Journal. 2011. № 4. P. 95-99.

5. Tikhanov VI, Shabanov PD Pharmacological analysis of the change in the content of di-new conjugation, methyl esters of fatty acids of liver lipid fractions by holinotropic agents against the background of supercooling of rats // Reviews of clinical pharmacology and drug therapy. 2017. T.15, No. 1, p. 33-40.

6. Feoktistova NA, Grigoriev NR, Borodin EA Influence of diet with the predominance of soybean on the cognitive abilities of rats // Far Eastern Medical Journal, 2017.-? 1.-P.70-74.

7. Borodin E.A., Menshikova I.G., Dorovskikh V.A. et all. Effects of soy protein isolate and casein on blood lipids and glucose in Russian adults with moderate hyperlipidemia // J. Nutr. Sci. Vit. 2009, 50, 6: 492-497.

8. Borodin E.A., Menshikova I.G., Dorovskikh V.A. et all. Antioxidant and Hypocholester-olemic Effects of Soy Foods and Cardiovascular Disease. // Soybean and Health. (Ed.by Hany A. El-Shemy). In-Tech, Croatia, 2011, p. 407-424.

9. Borodin E.A., Shtarberg M.A., Prikhodko A.G. et all. Modified noninvasive method of study of the oxidation of lipids of airways // Der Pharma Chemica. 2015, 7, 11:186-192.

10. Cocchi M., Minuto C., Tonello L. et al. Linoleic acid: Is this the key that unlocks the quan-tum brain? Insights linking broken symmetries in molecular biology, mood disorders and personalistic emergentism // BMC Neurosci., 2017, 18:38.

11. Fedeli E., Jacini G. Lipid composition of vegetable oils // Adv Lipid Res. 1971; 9:335-82

12. Folch J., Lees M., Sloan Stanley G.H. A simple method for the isolation and purification of total lipides from animal tissues // J Biol Chem. 1957, 226(1):497-509.

13. Fournier L.R., Ryan Borchers T.A., Robison L.M., et.all. The effects of soy milk and isofla-vone supplements on cognitive performance in heslthy postmenopausal women // J. Nutr Health Aging. 2007, 11(2):155-64.

14. Ivanov D., Levic J.D., Sredanovic S.A. Fatty acid composition of various soybean products // Food and Feed Research. 2010, 2: 65-70.

15. Katayama S., Imai R., Sugiyama H., et.all. Oral administration of soy peptides suppresses cognitive decline by induction of neurotrophic factors in SAMP8 maice // J Agric Food Chem. 2014 Apr 23;62(16):3563-9.

16. Kaufman P.B., Duke J.A., Brielmann H., et all. A comparative survey of leguminous plants as sources of the isoflavones, genistein and daidzein: implications for human nutrition and health // J Altern Complement Med. 1997. 3 (1): 7-12.

17. Martinez M. and Mougan I. Fatty Acid Composition of Human Brain Phospholipids During Normal Development // J. Neurochem. 1998, 71: 2528-2533. 
19. United States Department of Agriculture, Agricultural Statistics 2004. Table 3-51

20. Zhan S., Ho S.C. Meta-analysis of the effects of soy protein containing isoflavones on the lipid profile // Am J Clin Nutr 2005, 81: 397- 408

\title{
DOI 10.22448/AMJ.2017.3.34-35 DISCUSSION ON THE THEORY OF WUII POWDER IN TREATING UTERINE FIBROIDS
}

\author{
Fu Qiang1, Hu Xiaoyang2, Pang Yuhang1 \\ 1.Heilongjiang University of Traditional Chinese Medicine,Haerbin,China 2. First Affiliated Hospital Heilongjiang Univer- \\ sity of Chinese Medicine, Haerbin, China
}

Abstract: uterine myoma is the most common benign tumor of female reproductive organs, the prevalence of up to $51.2 \% \sim 60 \%$. The etiology of this disease is unclear, is difficult to cure the disease of department of gynaecology. Five jisan as treatments for cold, wet, qi, blood, phlegm, five kinds of stagnants, based on the theory of traditional Chinese medicine for five jisan theorizing about the treatment of uterine fibroids.

Key words: Wuji Powder; uterine fibroids; abdominal mass

Uterine fibroids are the most common benign tumors in women's reproductive health. They are prone to 30-50 year old women. Uterine fibroidsnot as malignant as a direct harm to the lives of patients, but the symptoms caused by a serious threat to women's health.There is no name of uterine fibroids in traditional Chinese medicine(TCM), but according to its clinical manifestations should be attributed to the "Zhengjia" ,"stone Zhengjia "and other areas[1].Wuji Powder is recorded in <Prescriptions People's Welfare Pharmacy>, which is treat with cold, wet, qi, blood and phlegm. This article will discuss from the Zhengjia etiology and pathogenesis and the composition and compatibility of the Wuji Powder.

\section{Knowledge of uterine fibroids in TCM}

According to the clinical symptoms: abdominal mass, menstrual extension, the amount of excessive, abdominal pain and other symptoms, will be attributed to the "tumor" category. <Ling Shu>:"Stone addiction was born in the womb, the cold qi in the sub-door, sub-door occlusion, qi can not pass, blood stasis shrimp to flow, days to benefits, like have a baby....",<Lady's prescription>:"The abdomen of the woman's blood, by the menstrual closure, or postpartum blood is not complete, or cold and stagnant, for a long time without the accumulation of scar Zheng Jia carry on."Blood chamber is open, uterine emptiness, wind cold dampness evil virtual invasion of the uterus, stagnation of blood, cold condensate blood stasis; or intercourse section, damage the blood cells, or worry about spleen, or Yu injury liver, can lead to blood stasis, tied to the cells, gradually become blocks, increasingly large uterine fibroids. [2]<Fuke Yuchi> emphasize the relationship between emotional abnormality and morbidity.In short, blood stasis is the pathogenesis of fibroids critical pathogenesis, or qi stagnation, or phlegm stasis type, or Qi and blood stasis. Disease occurs in the process, often dirty organs dysfunction, especially in the liver, spleen, kidney dysfunction more common.

\section{The group analysis of Wuji Powder}

Wuji Powder scattered from the <Prescriptions People's Welfare Pharmacy>, composed by the Atractylodes, Magnolia, dried tangerine peel, Pinellia, Poria, licorice, ephedra, cassia twig, white peony root, angelica, Chuanxiong, dried ginger, Citrus aurantium, Angelica and other 15-drug.Atractylodes, Magnolia, dried tangerine peel, licorice is Pingwei Powder for the transport of spleen dampness digestion. Dried tangerine peel, Pinellia, Poria, licorice as the main phlegm of the Erchen Tang.There is governance of the sun table card Guizhi soup; another rule of phlegm Ling Guigu Gan Tang; kidney disease Ling Jiang Gan Gan Tang.Siwu decoction to remove Rehmannia, with the blood of the meridian of the power; there ephedra Guixi Xin Wen published to scatter cold;Dried ginger, Guizhi, Citrus aurantium, Magnolia temperature in the qi, Dried tangerine peel, Pinellia ternata and ephedra, Campanulaceae Xuanfei phlegm; Ephedra, cassia twig, dried ginger, white peony root, angelica, licorice with the meaning of the side of the soup, all drugs combined, can eliminate the table, in the gas, blood cold wet.[3]Because this can cure cold, wet, qi, blood, sputum five kinds of weakness and the dosage form and powder mainly to take "scattered people also scattered" effect, named Wuji powder.

\section{Theoretical Analysis of Wuji Powder in Treating Uterine}

Uterine fibroids Zhengjia common gynecological diseases, the main cause of qi stagnation, blood stasis and phlegm pattern three causes. The main pathogenesis of the viscera and bwels dysfunction for the woman, resulting in qi deficiency with blood stasis, and phlegm-dampness generated, and then accumulate in the belly, in the course of the disease, the three each other cause and effect, mutual transformation, complex. In the past, treatment is often based on activate blood and resolve stasis, and the disease at different stages of its etiology is not the same, and the disease often changes in the pathogenesis, if the prescription medication can not take care of both, it is difficult to achieve therapeutic effect. [4] The disease due to cold in the middle form dampness, dampness agglomerate is phlegm, phlegm-dampness caused qi stagnation and blood stasis.So the treatment is used to activate blood and resolve stasis, move qi to relieve pain, warm the meridian to dissipate cold,fortify the spleen and drain dampness. Until remove the cold, resolve phlegm-dampness, spleen yang back to the revitalization,harmony of qi and blood, the card that is cured.In the Wuji Powder, Ephedra, Angelica promote sweating to release the exterior, Herb, Chuan Park, dried tangerine peel, Zhigancao dry dampness to 34 Амурский медицинский журнал №3 (19) 2017 